\title{
Enhanced Transmission Power Control Mechanism based on RSSI for MANET
}

\author{
Dinesh Ratan Gautam \\ School of Information \\ Technology, RGPV Bhopal
}

\author{
Sanjeev Sharma \\ School of Information \\ Technology, RGPV Bhopal
}

\author{
Santosh Sahu \\ School of Information \\ Technology, RGPV Bhopal
}

\begin{abstract}
Today's power consumption is the critical issue in MANET. Since, we have limited battery power to operate nodes. Due to the lack of power (Energy), the nodes "die out" from the network. Recently, power control in mobile ad hoc networks has been the focus of extensive research. Energy of nodes is consumed according to transmission power required by the radios. In this paper, we develop a mechanism, Enhanced Transmission Power Control Mechanism (ETPCM) that minimizes the required transmission power consumption of radio during packet transmission. In this work, the transmission power is dynamically set according to the distance and the distance can be calculated by using a parameter Receiving Signal Strength Indicator (RSSI) between these nodes. IEEE $802.11 \mathrm{~b}$ is a standard that specifies the physical layer and media access control designed for low-rate wireless local area networks (WLAN), it is used as radio at PHY layer (PHY802.11b) and protocol at MAC layer (MAC802.11), and it is generally used for MANET.
\end{abstract}

\section{Keywords}

Transmission Power, RSSI, IEEE 802.11b, ETPCM.

\section{INTRODUCTION}

MANET [1] is a collection of mobile nodes without any predefined and centralized infrastructure. Nodes are mobile and fixed in MANET. To communicate these nodes are use wireless medium. These nodes can be arbitrarily located and are free to move randomly at any given time, thus allowing network topology and interconnections between nodes to change rapidly and unpredictably. Node mobility can vary from almost stationary to constantly moving nodes. Two important issues are getting the attention of research area in MANET. First is power management and second is routing. To search the route between source and destination there are many routing protocols are used like DSR, AODV and DSDV etc. Some of routing protocols are also used for power management, like PARO (Power Aware Routing Optimization) [2]. Power requirement of the node define at the time of transmission, which is required by the radio. The power is consumed in node at the time of routing, transmission, receiving and processing the packets of data. The nodes are mobile in MANET. Nodes are battery operated devices. It is not possible to change the battery at the time of working. Battery power is limited in the node. The path established by using routing protocol between the nodes. When the path established, nodes start the communication. If the battery power is low then that node die out from the path. Routing protocol again start route discovery process to find another route. Many organizations work for increase the battery life of nodes by reducing the waste power.

Radios in communication system consume a fixed amount of transmission power to transmit packets between nodes either they are near of enough far to each other. In this dissertation work we use PHY $802.11 \mathrm{~b}$ as radio in wireless communication system.

\section{IEEE 802.11b}

IEEE 802.11b [3] is an industry standard technology for wireless LAN (WLAN) communication, with a purpose to provide network flexibility, low cost and low power consumption. This standard is suitable for applications which requiring low datarate communications in an ad hoc self organizing network [4]. $802.11 \mathrm{~b}$ uses radio wave for communication rather than wired cable infrastructure. Wireless LANs are usually implemented as the final link between the existing wired network and a group of client computers. The Institute of Electrical and Electronics Engineers (IEEE) created the original 802.11 specification in 1997 as the standard for wireless LANs [5]. 802.11 will work with $1 \mathrm{Mbps}$ and $2 \mathrm{Mbps}$ data rates. The most critical aspect with 802.11 is limited throughput. This standard is to slow to support the general requirements. After analysis of 802.11 standard Institute of Electrical and Electronics Engineers (IEEE) created a new standard for providing high data rate up to $11 \mathrm{Mbps}$ called 802.11 b. It is also known as 802.11 high rate. With 802.11 b WLANs, mobile users can get Ethernet levels of performance, throughput, and availability. The architecture of $802.11 \mathrm{~b}$ is similar to the 802.11 , but 802.11 b shows affects only the physical layer, adding high data rates in the OSI model.

802.11b standard create to support two new speeds (5.5 Mbps and $11 \mathrm{Mbps}$ ) [5]. The implication with $802.11 \mathrm{~b}$ is that it can work with the direct-sequence spread spectrum (DSSS), but it cannot work with the Frequency-hopping spread spectrum (FHSS) at the physical layer, because the physical layer cannot support frequency hopping at high speed. To encode all the data, original 802.11 specifies 11-bit chipping which is called Baker sequence. Each 11-chip sequence represents a single data bit (1 or 0 ), and is converted to a waveform, called a symbol, that can be sent over the air. These symbols are transmitted at a $1 \mathrm{MSps}$ (1 million symbols per second) symbol rate using a technique called Binary Phase Shift Keying (BPSK) [6]. In the case of 2 Mbps, a more sophisticated implementation called Quadrature Phase Shift Keying (QPSK) [7] is used; it doubles the data rate available in BPSK, via improved efficiency in the use of the radio bandwidth. 
802.11b standard uses advance coding technique rather than Baker Sequence to increase the data rate. It specifies Complementary Code Keying (CCK) [8], which consists of a set of 64 eight-bit code words. The $5.5 \mathrm{Mbps}$ rate uses CCK to encode 4 bits per carrier, while the $11 \mathrm{Mbps}$ rate encodes 8 bits per carrier. Both speeds use QPSK as the modulation technique and signal at $1.375 \mathrm{MSps}$. This is how the higher data rates are obtained. Table 1 shows the differences.

Table 1. 802.11b Data Rate Specifications

\begin{tabular}{|c|c|c|c|c|}
\hline $\begin{array}{c}\text { Data } \\
\text { Rate }\end{array}$ & $\begin{array}{c}\text { Code } \\
\text { Length }\end{array}$ & $\begin{array}{c}\text { Modul- } \\
\text { ation }\end{array}$ & $\begin{array}{c}\text { Symbo-I } \\
\text { Rate }\end{array}$ & $\begin{array}{c}\text { Bits/Sy- } \\
\text { mbol }\end{array}$ \\
\hline $1 \mathrm{Mbps}$ & $\begin{array}{c}11 \text { (Barker } \\
\text { Sequence) }\end{array}$ & BPSK & 1 MSps & 1 \\
\hline $2 \mathrm{Mbps}$ & $\begin{array}{c}11 \text { (Barker } \\
\text { Sequence) }\end{array}$ & QPSK & $1 \mathrm{MSps}$ & 2 \\
\hline $\begin{array}{c}\text { Mbps } \\
11\end{array}$ & $8(\mathrm{CCK})$ & QPSK & $\begin{array}{c}1.375 \\
\text { MSps }\end{array}$ & 4 \\
\hline \begin{tabular}{c} 
Mbps \\
\hline$b(\mathrm{CCK})$
\end{tabular} & QPSK & $\begin{array}{c}1.375 \\
\text { MSps }\end{array}$ & 8 \\
\hline
\end{tabular}

$802.11 \mathrm{~b}$ uses dynamic rate shifting. It allows data rate adjust automatically with changing nature of radio. Ideally, user connect at full $11 \mathrm{mbps}$ rate, but the user moves beyond the optimal range of $11 \mathrm{mbps}$ it will $802.11 \mathrm{~b}$ devices will transmit at lower speeds, falling back to 5.5, 2, and $1 \mathrm{Mbps}$.

\section{ENERGY SAVING AND WASTE IN WIRELESS NETWORK}

Energy saving will be done at different layer of wireless network's architecture [9]. There are different methods are used to save power at different layers. The node doesn't need high bandwidth but it requires low latency and low power consumption in wireless network. In this section we will describe two level of energy saving. These are Device level and network level.

\subsection{Energy saving at node level}

Each node has at least two energy levels Emax and Emin. Former is the power required to reaches farthest node while latter is the power required to reach the nearest node. . It is assumes that there may exist some intermediate power level between Emax and Emin. Let $\mathrm{E}(x y)$ be the power needed to support communication from node $\mathrm{x}$ to $\mathrm{y}$, we called it symmetric if $\mathrm{E}(x y)=\mathrm{E}(y x)$ [10]. $\operatorname{Emax}(x)$ and $\operatorname{Emin}(x)$ are the maximum and minimum power level of node $\mathrm{x}$. When $\mathrm{E}(x y) \geq \operatorname{Emin}(x)$ and $\mathrm{E}(x y) \leq \operatorname{Emax}(x)$ [10], then communication between $\mathrm{u}$ and $\mathrm{v}$ is possible otherwise it is not possible.

\subsection{Energy Saving at MAC Layer}

To establish the coordination between nodes MAC layer is responsible. When a radio signal send or listen to the communication channel it uses large amount of node's power.
Nodes communicate with each other within a wireless channel in wireless network. In communication at least two nodes can access one communication channel at same time; this is the region to collision occurrence between nodes. The collision is responsible for packet conjunction, energy consumption, and retransmission. Retransmission creates waste of energy. These factors affect the networks life time. To avoid collision between nodes is the most important responsibility for the MAC layer. The Mac layer provide free channel for communication and avoid the collisions by using CSMA/CA (Carrier Sense Multiple Access with Collision avoidance) [11].

CSMA/CA is a modification of CSMA. Collision avoidance is used to improve the performance of CSMA by attempting to be less "greedy" on the channel. If the channel is sensed busy before transmission then the transmission is deferred for a "random" interval. This reduces the probability of collisions on the channel.

\subsection{Energy Saving at Network Layer}

There are many proposals which try to define an energy efficient routing protocol, capable of routing data over the network and of saving the battery power of mobile nodes. To make more efficient routing protocols, we can add energy aware functionalities in existing routing protocol (like AODV, DSR etc.). The aim of energy-aware routing protocols is to reduce energy consumption in transmission of packets between a source and a destination, to avoid routing of packets through nodes with low residual energy, to optimize flooding of routing information over the network and to avoid interference and medium collisions. By using the energy aware metrics we can make more energy efficient routing protocols.

The first energy-efficient routing is MTPR (Minimum Transmission Power Routing) [12]. Its mechanism uses simple energy metric, to represent total energy consumption in transmission. MTPR reduces total transmission power consumed per packet. Another routing metric is MBCR (Minimum Battery Cost Routing) [12]. The proposed battery cost function is:

$$
f i(t)=1 / c i(t)
$$

Where $c i(t)$ is the battery capacity of node $n i$ at time $t$. The less capacity a node has, the more reluctant it is to forward packets.

\subsection{Energy waste in wireless network}

Nodes have limited energy to store so we have needed to save the waste power of node. In $802.11 \mathrm{~b}$ standard have following regions for power waste

\subsubsection{Idle sensing}

When node listens an idle channel to receive possible traffic. When the node does not know when it will receive packet that node must keep its radio ready to receive mode, which consume so much energy?

\subsubsection{Collision}

To establish communication between nodes at least two nodes must share the channel at same time. When nodes start transmission of packets the collision is occur and packet dropped. Node retransmits that packet which consumes energy again. 


\subsubsection{Overhearing}

When a node receives and decodes packets that are distinct for other node. It produces unnecessary packets. These packets are major cause of energy waste.

\section{PROPOSED WORK}

Energy saving is an important factor for wireless ad hoc network. To save the energy of node we proposed a mechanism called ETPCM (Enhanced Transmission Power Control Mechanism), which is based on RSSI (Receiving Signal Strength Indicator) [13] and distance measurement between nodes. In ETPCM we modify the working process of radio $802.11 \mathrm{~b}$ to save the transmission power. As we know the transmission power is depend on required transmission power for used radio in data transmission. In ETPCM we use a parameter RSSI to change the static transmission power into dynamic transmission power.

\subsection{Principal of RSSI}

The principal of RSSI describes relationship between transmitted power and received power of wire-less signal and the distance among nodes. This relationship has shown by an equation (1) [14].

$$
P_{r}=P_{t}^{*}(1 / d)^{n}
$$

Where $\operatorname{Pr}$ is receiving power, $P t$ is the transmitted power; $d$ is the distance between sender and receiver node and $n$ is the transmission factor whose value depends on the propagation environment. Take 10 times the logarithm of both sides on (1), and then Equation (1) is transformed to Equation (2).

$$
10 * \log P_{r}=10 * \log P_{t}-10 n * \log d
$$

$P_{r}$, the transmitted power of nodes, are given. $10 \log P$ is the expression of the power converted to $\mathrm{dBm}$. Equation (2) can be directly written as Equation (3).

$$
\operatorname{Pr}(\mathrm{dBm})=A-10 * \log d
$$

$\mathrm{A}$, is received power from references distance which is 1 meter. By Equation (3), we can see that the values of parameter A and parameter $\mathrm{n}$ determine the relationship between the strength of received signals and the distance of signal transmission.

\subsection{Relationship between RSSI and distance}

Power decreases logarithmically with distance. The average loss for a given distance is expressed using a Path Loss Exponent. For calculating the received power based on this model, we first calculate the received power at a reference distance using the Friis formula (given in equation 1). Then, we incorporate the effect of path loss exponent and shadowing parameters [15].

$$
\text { RSSI }=-\left(10 * \log _{10}\left(\mathrm{~d}_{\mathrm{ij}}\right)-\mathrm{A}\right)
$$

The theoretical distance between nodes is given by

$$
\mathrm{d}_{\mathrm{ij}}=10^{\frac{\mathrm{RSSI}-\mathrm{A}}{-10 * \mathrm{n}}}
$$

In practical application environment, path loss model having problem with multi-diameters, diffraction, obstacle and so have an impact on the wireless signal transmission [16].However it is indispensable to adjust $\mathrm{A}$ and $\mathrm{n}$, the model according to specific environment.

\subsection{Proposed ETPCM}

In my proposed work, Enhanced Transmission Power control Mechanism for radio PHY802.11b based on RSSI and Distance for MANET (ETPCM), we make Four Levels of calculated distance by using RSSI and four Levels of Transmission Power. We set transmission power according to coordination value of calculated distance. My implementation work is on packet receiving process of radio $802.11 \mathrm{~b}$, when transmitter and receiver are ready to process.

\subsection{Algorithm Design}

1. Calculate RSSI for each node;

2. Calculate distance between nodes by using formula:

$$
\mathrm{d}_{\mathrm{ij}}=10^{\frac{\mathrm{RSSI}-\mathrm{A}}{-10 * \mathrm{n}}}
$$

\section{3. if ((mode $==$ PHY_RECEIVING)} $\& \&($ ReceiveMsg $==$ TransmitMsg $))$

\{

If (Distance $>=100)\{$

Then set $\mathrm{Tx}=15 \mathrm{dBm} ;$ \}

else if (Distance $<100$ and Distance $>=70)\{$

Then set $\mathrm{Tx}=14 \mathrm{dBm}$;

else if (Distance $<70$ and Distance $>=50)\{$

Then set $\mathrm{Tx}=13 \mathrm{dBm}$; \}

else \{

Then set $\mathrm{Tx}=12 \mathrm{dBm} ;$ \}

\}

Where,

RSSI= Receiving Signal Strength Indicator value.

$\mathrm{dij}=$ distance between node $\mathrm{I}$ and node $\mathrm{j}$.

$\mathrm{n}=2$ (for free space ground model).

$A=-32(\mathrm{dBm})$ receiving signal strength between two nodes at 1 metre.

$\mathrm{Tx}=$ Transmission Power in $\mathrm{dBm}$

\section{SIMULATION METHODOLOGY}

In this work, we use QualNet 5.0 [17] simulator for simulation. Initially total numbers of nodes are 10 for various pause time and we also vary number of nodes to $5,10,15,20$, and 25 at fixed pause time. Its value is 60 s. Simulation time is taken 180 seconds and seed as 1 . All the scenarios have been designed in $100 \mathrm{~m} \mathrm{x}$ $100 \mathrm{~m}$ area. Mobility model used is Random Way Point (RWP) 
and Energy Model used is Generic Model. In this mobility model a mobile node is initially placed in a random location in the simulation area, and then moved in a randomly chosen direction between at constant speed. We set transmission data rate at 6 mbps. We choose fixed Mobility speed $=10 \mathrm{~m} / \mathrm{s}$, and varies pause time to $00,15,30,45$, and $60 \mathrm{~s}$. The initial transmission power is used $15 \mathrm{dBm}$ (default transmission power of radio PHY802.11b). All the simulation work was carried out using propagation model free space and constant shadowing model with AODV [1] routing protocol.

\section{PERFORMANCE METRICS USED}

The following four metrics have been chosen to compare the propagation models:

6.1 Throughput is the measure of the number of packets successfully transmitted to their final destination per unit time. It is the ratio between the numbers of sent packets vs. received packets.

6.2 Average End to End Delay End-to-end delay indicates how long it took for a packet to travel from the CBR source to the application layer of the destination. It represents the average data delay an application or a user experiences when transmitting data.

6.3 Average Jitter Effect signifies the Packets from the source will reach the destination with different delays. A packet's delay varies with its position in the queues of the routers along the path between source and destination and this position can vary unpredictably.

6.4 Transmission Power is the amount of Power which is used to transmit signal by node.

6.5 Energy Consumption Total energy consumption, which required for transmission, receiving and processing signal by node.

\section{RESULTS AND ANALYSIS}

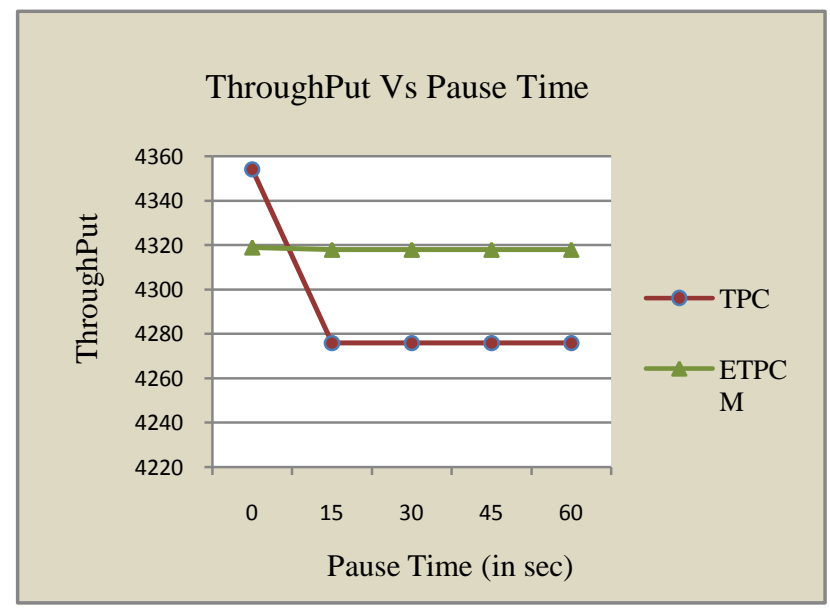

Fig: 1

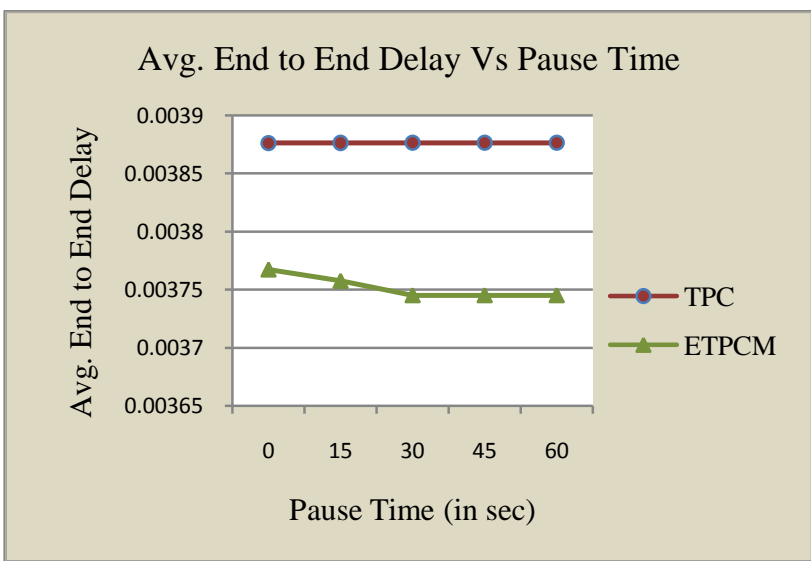

Fig: 2

Figure 1 and 2 shows throughput and average end to end delay respectively with different variation in pause time. It observes at pause time 0, the throughput of ETPCM is less. When we increase the pause time the through put will greater than the values of TPC. The average end to end delay is less at every pause time.

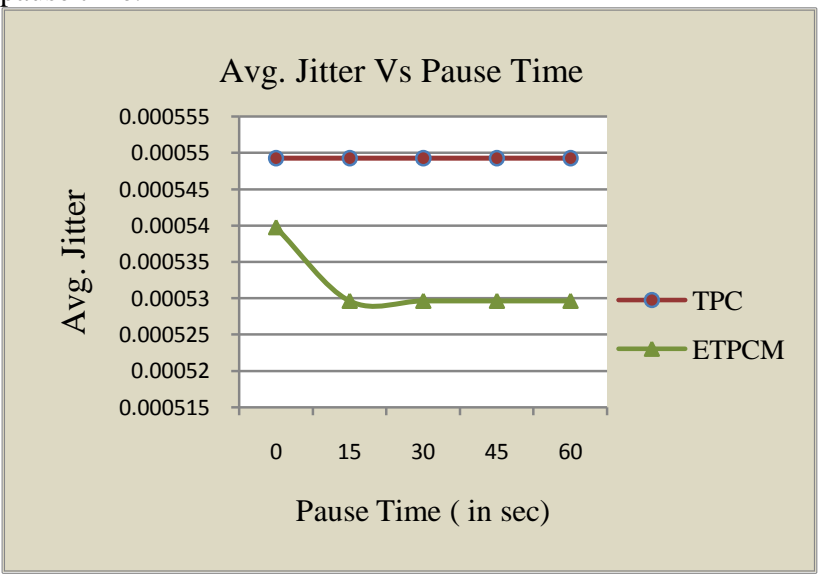

Fig: 3

Figure 3 shows that average jitter with different variation in pause time. It observes at all pause time the value of ETPCM is always less than values of TPC. It means the performance of ETPCM is better than TPC.

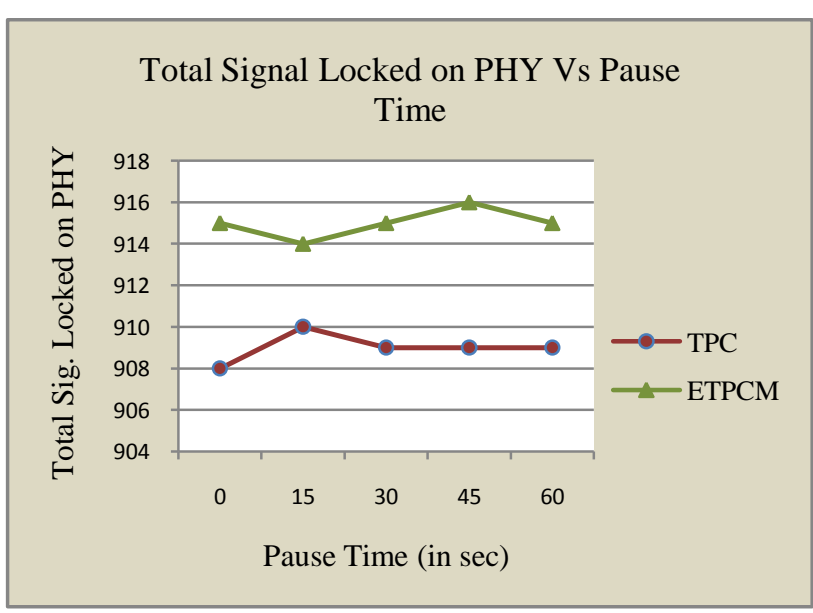

Fig: 4 


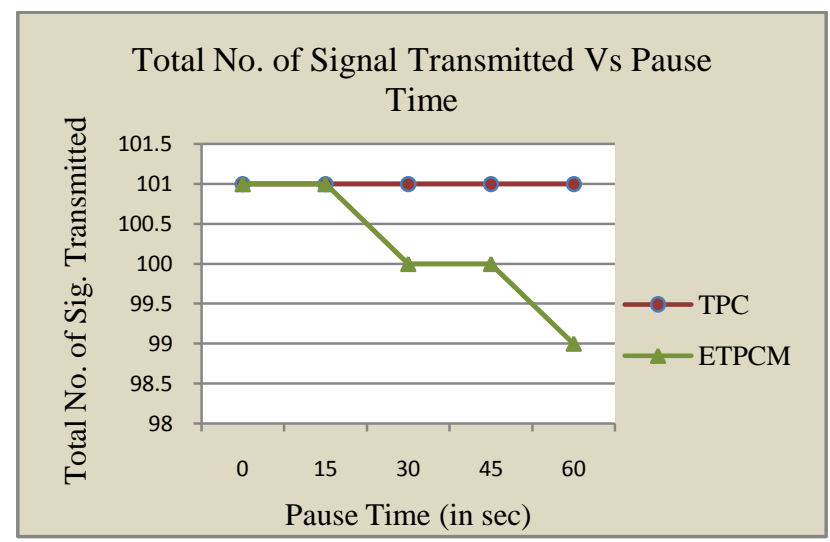

Fig: 5

Figure 4 and 5 shows that total signal locked on physical layer and total number of transmitted signal respectively with different variation in pause time. ETPCM is better performing than simple TPC with total signal locking at physical layer. In figure 5 total number of signal transmission is reduces in ETPCM. For this parameter, the proposed ETPCM reduces its performance than simple TPC.

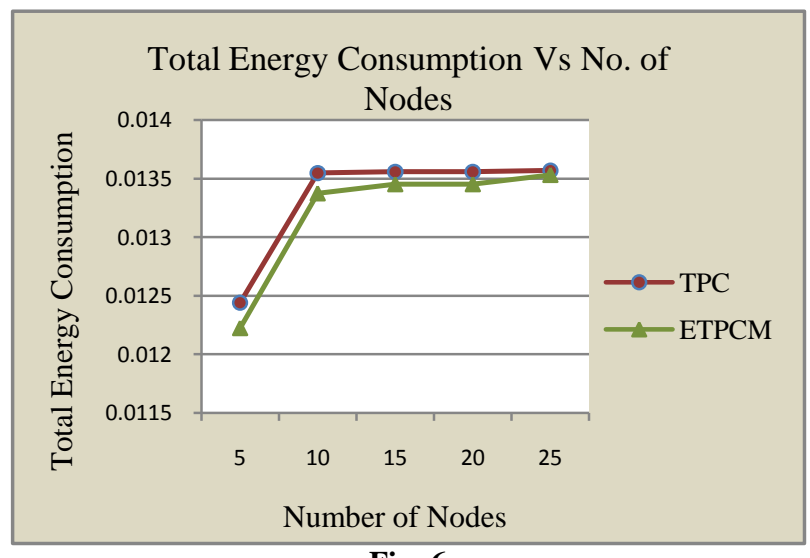

Fig: 6

Figure 6 shows total energy consumption with different variation in number of nodes. ETPCM can consume less energy than simple TPC. When we use ETPCM the nodes can use less power and increase the battery life of node. When the number of nodes increases then the power consumption is also increases.

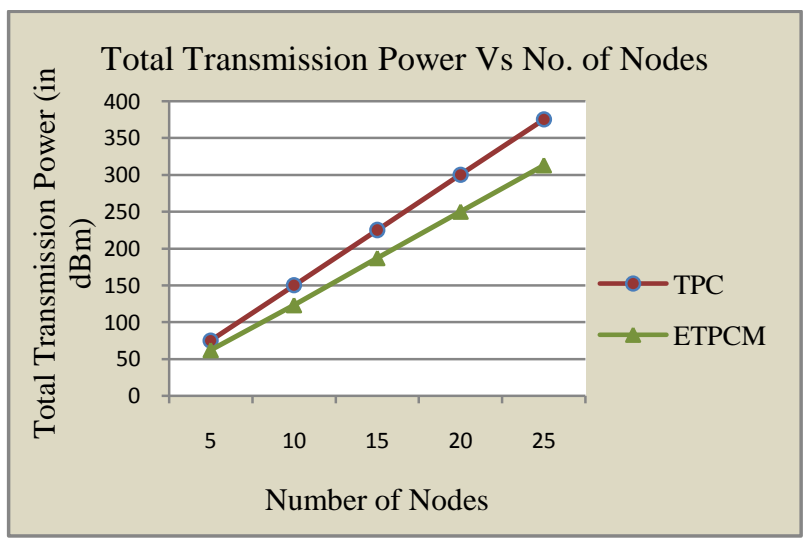

Fig: 7
Figure 7 shows that total required transmission power with variation in number of nodes. When we increase the number of nodes the transmission power is also increased, but in this figure ETPCM consume less power than simple TPC in transmission of packets.

\section{CONCLUSION AND FUTURE WORK}

In the wireless network each and every node required power for transmit signals. Proposed ETPCM mechanism is mainly based on transmission power conservation of radio PHY802.11b based on a parameter Receiving Signal Strength Indicator (RSSI) and Distance. We set the required transmission power of radio PHY 802.11b based on calculated distance which can be calculate by RSSI. ETPCM is save power consumption and increase the life time of nodes as well as network. ETPCM is mainly design for transmission power control of radio PHY 802.11b. The result and analysis shows that ETPCM is working as for design and presents better performance than simple TPC. All results and analysis, given in section 7, show that ETPCM performs better than simple TPC.

As part of our future work we simulate ETPCM mechanism with varying no. of Nodes, simulation time and different scenarios like battle field, urban areas etc. this work also can perform with different networks like sensor network (IEEE 802.15.4). Different mobility models will be considered for the movement of the nodes to determine receiving signal strength and distance between nodes and other parameter as mobility metric across a range of mobility models. One also can represent this Enhanced Transmission Power Control Mechanism (ETPCM) with addition of Interference Power Control Management (IPCM) to save interference power consumption with transmission power.

\section{REFERANCES}

[1] Charles E.Perkins, "Ad hoc Networking", Addison Wesley, 2001.

[2] Javier Gomez, Andrew T. Campbell, "PARO: Supporting Dynamic Power Controlled Routing in Wireless Ad Hoc Networks" Wireless Networks 9, 443-460, 2003 Kluwer Academic Publishers, Manufactured in The Netherlands.

[3] Arindam Ghosh, Aboubaker Lasebae, Enver Ever "Performance Evaluation of Wireless IEEE 802.11(b) used for Ad-Hoc Networks in an ELearning Classroom Network", Middlesex University, the Burroughs, Hendon, London NW4 4BT.

[4] Falko Dressler, "Self-Organization in Ad Hoc Networks: Overview and Classification", Autonomic Networking Group, Dept. of Computer Science 7, University of Erlangen, Martensstr. 3, 91058 Erlangen, Germany.

[5] Plamen Nedeltchev, Felicia Brych, "Wireless Local Area Networks and the 802.11 Standard", PhD Thesis report, IIT Mumbai and Internet Draft 2002.

[6] Ryan Reed "Implementation of a BPSK Transceiver for use with the University of Kansas Agile Radio", Bachelor of Science, University of Kansas, Lawrence, Kansas, 2004.

[7] Amit P. Jardosh, Krishna N. Ramachandran, Kevin C. Almeroth, Elizabeth M. Belding-Royer "Understanding 
link-layer behavior in highly congested IEEE $802.11 \mathrm{~b}$ wireless networks" ACM NewYork, NY, USA (C2005.

[8] Javier del Prado Pavon, Sunghyun Choi "Link Adaptation Strategy for IEEE 802.11 WLAN via Received Signal Strength Measurement" Communications, 2003. ICC '03. IEEE International Conference on.

[9] Matthew B. Shoemake, "Wi-Fi (IEEE 802.11b) and Bluetooth Coexistence Issues and Solutions for the $2.4 \mathrm{GHz}$ ISM Band", $\mathrm{PhD}$ Thesis at University of California.

[10] Niranjan Kumar Ray, Ashok Kumar Turuk, "Energy Efficient Techniques for Wireless Ad Hoc Network", proceeding of first international joint conference on information and communication technology, IJcICT 2010, Pages 105-111, January, 2010.

[11] Duong Hoang, Ronald A. Iltis, "Performance Evaluation of Multi-hop CSMA/CA Networks in Fading Environments", Department of Electrical and Computer Engineering University of California, Santa Barbara, CA 93106-9560.

[12] Morteza Maleki, Karthik Dantu, and Massoud Pedram, "Power-aware Source Routing Protocol for Mobile Ad Hoc
Networks", Dept. of EE-Systems, University of Southern California, Los Angeles, CA 90089.

[13] Charalampos Papamanthou, Franco P. Preparata , Roberto Tamassia, "Algorithms for Location Estimation Based on RSSI Sampling ”, S. Packet (Ed.), ALGOSENSERS 2008, LNCS 5389, pp. 72-86, 2008. c Springer-Vertag Berlin Heidelberg. 2008.

[14] Zhang Jianwu, Zhang $\mathrm{Lu}$ "Research on Distance Measurement Based on RSSI of ZigBee" CCCM IEEE 2009.

[15] Neeraj Choudhary, Ajay K Sharma "Performance Evaluation of LR-WPAN for different Path-Loss Models" International Journal of Computer Applications (0975 8887) Volume 7- No.10, October 2010.

[16] Torsten Muetze Patrick Stuedi Fabian Kuhn Gustavo Alonso "Understanding Radio Irregularity in Wireless Networks" 2007.

[17] Scalable Network Technology, "QualNet 5.0 simulator" tutorial and QualNet Forum, http://www.scalablenetworks.com/forums. 